\title{
Meningioma Induced Temporal Lobe Epilepsy; A Case Report
}

\author{
Hosein Delavar Kasmaei ${ }^{1}$; Fatemeh Shabani ${ }^{1,}$; Alireza Baratloo $^{2}$ \\ ${ }_{1}^{1}$ Department of Neurology, Shohadaye Tajrish Hospital, Shahid Beheshti University of Medical Sciences, Tehran, IR Iran \\ ${ }^{2}$ Department of Emergency Medicine, Shohadaye Tajrish Hospital, Shahid Beheshti University of Medical Sciences, Tehran, IR Iran \\ ${ }^{*}$ Corresponding author: Fatemeh Shabani, Department of Neurology, Shohadaye Tajrish Hospital, Shahid Beheshti University of Medical Sciences, Tehran, IR Iran. Tel:+98-9121579198, \\ Fax:+98-2122721155, E-mail:dr.fatemehshabani@gmail.com
}

Received: June 30, 2014; Accepted: August 19, 2014

\begin{abstract}
Introduction: Temporal lobe epilepsy (TLE) presented with behavioral and psychiatric disorders is one of the most hectic forms of epileptic seizures, in which a uniform pathology cannot explain its manifestations, and structural underlying causes should be kept in mind.

Case Presentation:This report described a 38-year-old female admitted to our department in emergency manner with episodic attacks of behavioral disorders and brief episodes of loss of consciousness each time accompanied with headache as a starting point, which lasted 5 to 10 minutes. Brain MRI showed a mass in the left temporal lobe finally diagnosed as a meningioma. After prescription of antiepileptic agents, headache, psychiatric disorders and hallucination attacks were stopped.

Discussion: Temporal lesions with behavioral changes can cause complicated and difficult cases. There is limited data about this, but it seems that psychiatric features of temporal lobe epilepsy could be controlled and treated easily only by antiepileptic agents.
\end{abstract}

Keywords:Meningioma; Psychiatric Disorder; Temporal Lobe Epilepsy

\section{Introduction}

Temporal lobe epilepsy (TLE) is one of the most hectic forms of epileptic seizures, which a uniform pathology cannot explain its manifestations and possibly accompanied by different underlying causes such as physiologies, treatment outcomes and even functional anatomies (1). Aggressive behaviors, psychosis and anxiety are frequent psychiatric manifestations observed in patients with TLE with different rates of occurrence. Psychiatric manifestations in epilepsy can appear concurrently with epilepsy improvement or abolition of epileptic activity (2). This case-report described a patient with meningioma in the left temporal lobe, which caused seizure with psychiatric presentation.

\section{Case Presentation}

A38-year-old female was admitted to this center following episodic changes in her behavior as agitation, anxiety and aggressive behaviors repeated 2-3 times a day for one year. She looked quite agitated and anxious, then began to scream and cry and had some aggressive behaviors. Furthermore, she complained severe headache during this time. Headache was localized in occiput region at first but generalized later. She had some hallucinations with expression of Lilliputian human, big spider, a woman with black clothes in each time. She did not remember these hallucinations after attacks. After 2-3 hours, she had a brief period of loss of consciousness for 5-10 minutes, as she was asleep and did not respond to others. Then she was confused for several minutes. After that she became conscious with relief of headache, hallucinations and behavioral changes. There was no family history of epilepsy. Neurological examinations had normal findings. Scalp EEG showed paroxysmal activity in the left temporal region. Brain MRI showed a round mass with definite margin, which was isointense on T1 and T2. This lesion had homogenous enhancement in brain MRI with gadolinium with dural tail suggesting meningioma (Figures 1, 2). After antiepileptic treatment with phenytoin, seizures were aborted. Her symptoms were improved. We continued the treatment by changing the medication to carbamazepine. Clinical response to this treatment was excellent. Moreover, she was admitted to neurosurgery department for resection of the tumor. The tumor was proved as meningioma by pathologic assessment. 

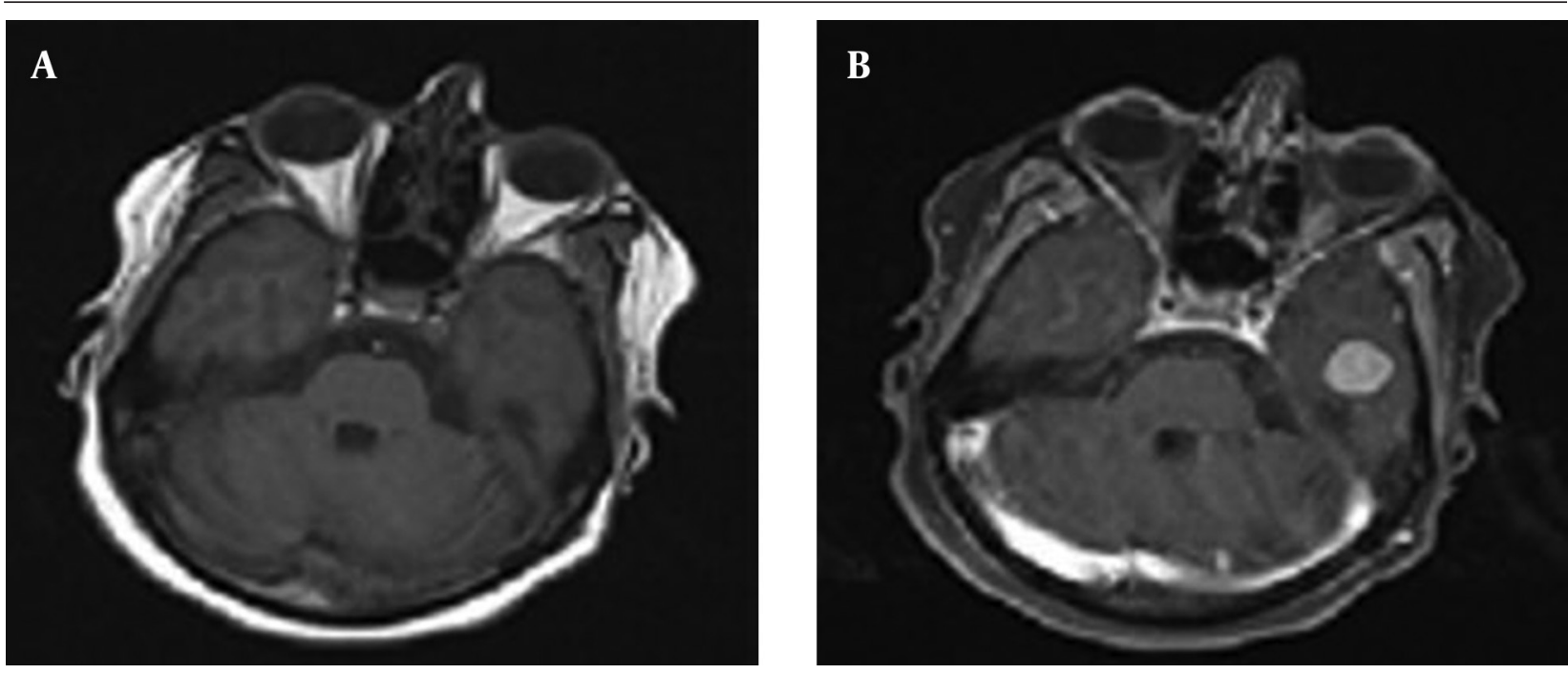

Figure 1. Axial Sequence of T1 Brain MRI Without Gadolinium (A) and With Gadolinium (B)
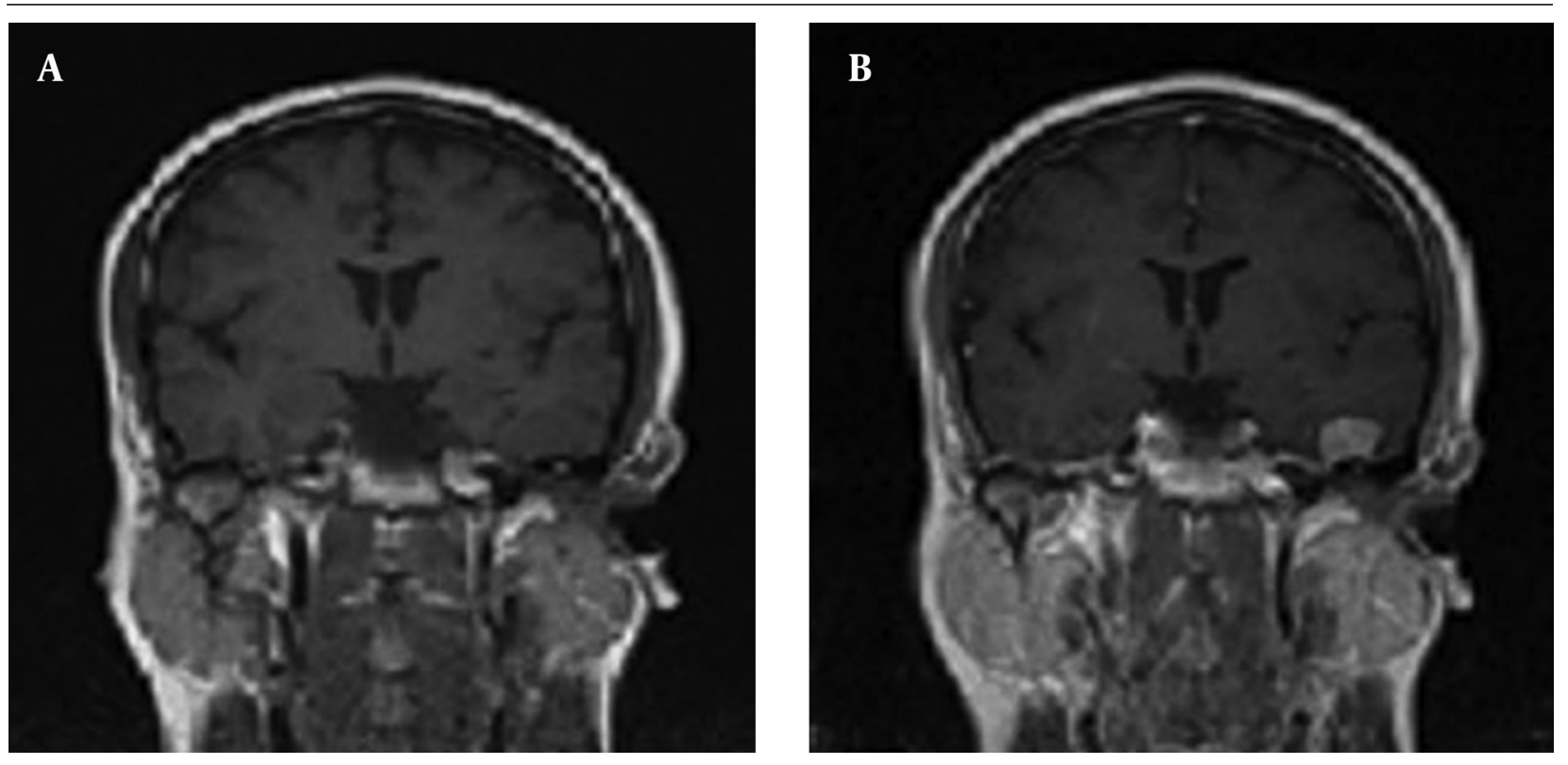

Figure 2. Coronal Sequence of T1 Brain MRI Without Gadolinium (A) and With Gadolinium (B)

\section{Discussion}

Meningioma is one of the most common brain masses originated from arachnoid cells, which can present with psychiatric symptoms such as anxiety disorders, depression, or even permanent personality changes in the absence of any apparent neurologic deficit (3). Rarely these manifestations can be suggestive of a kind of epilepsy called TLE. About 30 percent of patients with meningioma present with seizures and TLE is one of the uncommon types (4). On the other hand, the association between psychotic disorders as presentation of epilepsy has been controversial $(5,6)$. Manifestations related to each stage of a temporal lobe seizure may be helpful to determine both the localization and lateralization of seizure onset (7). Some researchers described the psychic phenomena as experiential hallucinations based on clinical observations and intraoperative stimulation studies $(8,9)$. Psychotic complaints in epilepsy are commonly seen but not particularly accompanied by TLE. Usually, clinical presentations of such disorders differ relevant to their temporal relation pertaining to seizure occurrence. Therefore, postictal symptoms of anxiety or psychosis differ in duration, severity and response to treatment with 
interictal symptomatology $(2,10)$. Routine neuroimaging to approach new onset psychiatric disorders is a controversy, but this case raises the importance of possible role of diagnostic studies in these patients (3). Temporal lesions with behavioral changes can cause complicated and difficult cases. There is limited data about this, but it seems that psychiatric features of temporal lobe epilepsy could be controlled and treated easily only by antiepileptic agents.

\section{Acknowledgements}

We would like to thank all staff of emergency and neurology departments who helped in diagnosis and management of this patient.

\section{Authors' Contributions}

All authors helped in study supervision, study concept and design, acquisition of data and drafting of the manuscript. Critical revision of the manuscript for important intellectual content was performed by Alireza Baratloo.

\section{References}

1. Thom M, Bertram EH. Temporal lobe epilepsy. Handb Clin Neurol.

\section{2;107:225-40}

2. Kanner AM. Recognition of the various expressions of anxiety, psychosis, and aggression in epilepsy. Epilepsia. 2004;45 Suppl 2:22-7.

3. Kumar T, Archana K, Carrol TL. Right Temporal Lobe Meningioma presenting as postpartum depression: A case report. Apollo Medicine . 2013;10(14):299-301.

4. Hamasaki T, Yamada K, Kuratsu J. Seizures as a presenting symptom in neurosurgical patients: a retrospective single-institution analysis. Clin Neurol Neurosurg. 2013;115(11):2336-40.

5. Assefa D, Haque FN, Wong AH. Case report: anxiety and fear in a patient with meningioma compressing the left amygdala. Neurocase. 2012;18(2):91-4.

6. D'Alessio L, Giagante B, Ibarra V, Papayannis C, Oddo S, Solis P, et al. [Analysis of psychotic disorders in patients with refractory partial epilepsy, psychiatric diagnoses and clinical aspects]. Actas Esp Psiquiatr. 2008;36(3):138-43.

7. Saint-Hilaire JM, Lee MA. Localizing and lateralizing value of epileptic symptoms in temporal lobe epilepsy. Can J Neurol Sci. 2000;27 Suppl 1:S1-5.

8. Pillai JA, Haut SR. Patients with epilepsy and psychogenic nonepileptic seizures: an inpatient video-EEG monitoring study. Seizure. 2012;21(1):24-7.

9. Coan AC, Cendes F. Understanding the spectrum of temporal lobe epilepsy: contributions for the development of individualized therapies. Expert Rev Neurother. 2013;13(12):1383-94.

10. Verhoeven WM, Egger JI, Gunning WB, Bevers M, de Pont BJ. Recurrent schizophrenia-like psychosis as first manifestation of epilepsy: a diagnostic challenge in neuropsychiatry. Neuropsychiatr Dis Treat. 2010;6:227-31. 